\title{
Growth Mechanism of (111)-Textured 3C-SiC Films on Si(100) Substrates
}

\author{
V. Radmilovic*; Di Gao**, R. Maboudian**, and U. Dahmen* \\ *National Center for Electron Microscopy, Lawrence Berkeley National Laboratory, and \\ **Department of Chemical Engineering, University of California, Berkeley, CA 94720
}

Heteroepitaxial growth of $3 \mathrm{C}-\mathrm{SiC}$ films on a $\mathrm{Si}(100)$ substrate can be achieved by chemical vapor deposition [1,2]. Under certain processing conditions, there is a transition from 3-D epitaxial islands to columnar grains, modifying the film texture from (100) to (111). This transition is more pronounced with increasing thickness and decreasing deposition temperature. The current work uses transmission electron microscopy to characterize the microstructure and investigate the mechanism of $3 \mathrm{C}-\mathrm{SiC}$ film growth on $\mathrm{Si}(100)$ substrates.

SiC films are deposited on $\mathrm{Si}(100)$ substrates by CVD using a single precursor DSB (1,3disilabutane, molecular structure $\mathrm{CH}_{3}-\mathrm{SiH}_{2}-\mathrm{CH}_{2}-\mathrm{SiH}_{3}$ ). The deposition begins by pressurizing the CVD reactor with $\mathrm{DSB}$ to approximately $5 \square 10^{-5}$ torr at a reactor temperature of $100^{\circ} \mathrm{C}$. The temperature is then increased to the ultimate growth temperature of $850^{\circ} \mathrm{C}$ at a rate of $50^{\circ} \mathrm{C} / \mathrm{min}$ at constant DSB pressure. The film growth rate under these conditions is about $150 \mathrm{~nm} / \mathrm{min}$. Conventional transmission electron microscopy was carried out in a JEOL 200CX microscope, operating at $200 \mathrm{kV}$, and high resolution microscopy in a CM300 FEG microscope operating at $300 \mathrm{kV}$.

We have found that the columnar grains (Fig. 1a) do not originate at the substrate surface but on a buffer layer (Fig. 2a) about 3 to $5 \mathrm{~nm}$ thick, consisting of interconnected 3D-islands (Fig. 2b), as proposed by Ernst and Pirouz [3]. The growth of epitaxial 3-D islands in a one-step deposition process using a single precursor has not been shown before. Initial nucleation of $\mathrm{SiC}$ grains occurs in parallel-cube orientation relationship at the interface with the substrate, and twinning is abundant even at the earliest stages of growth. It is apparent that growth is limited by the slow growth rate of the $\{111\}$ planes. From these observations, it is possible to understand the growth mechanism as follows. Initially, 3C-SiC grains nucleate epitaxially on the $\mathrm{Si}(100)$ substrate in a parallel-cube orientation relationship. The anisotropy in growth rate along $\{100\}$ and $\{111\}$ planes differs by at least a factor of $\sqrt{3}$, making $\{111\}$ faces the growth limiting form. However, twinning occurs frequently and appears to be associated with enhanced growth. Higher order twinning leads to new crystal orientations. During the growth competition following nucleation, the slower-growing grains are overgrown by faster-growing grains [4]. Growth will be most rapid in the directions that make the largest angle with the growth facets. This means that the grains that are aligned with their fastest growth direction normal to the substrate survive and become the $\{111\}$ oriented columnar grains we observe (Figs. $1 \mathrm{~b}$ and c). Our observations show that nucleation occurs in $\langle 100\rangle$ orientation (Fig. $2 b)$. Growth in this orientation will be limited until subsequent twinning generates new orientations that are closer to the $<111>$ growth direction favored by twinning. For example, first order twins will be oriented with their $<122>$ direction normal to the substrate, bringing a $<111>$ direction within $16^{\circ}$ of the substrate normal. Higher order twins can generate even more favorable orientations, until a $<111>$ direction is close enough to the substrate normal, within $\pm 9^{\circ}$, to allow uninhibited growth. Thus, as observed, the largest columnar grains are those that are closest to a $<111>$ orientation. This leads to the observed $<111>$ fiber texture.

\section{References}

[1] J.A. Powell, L.G. Matus and M.A. Kuczmarski, J. Electrochem Soc., 134 (1987) 1558.

[2] V. Cimalla, Th. Stauden, G. Eichhorn, J. Pezoldt, Mater. Sci. Eng. B, B61-62 (1999) 553.

[3] F. Ernst and P. Pirouz, J. Mater. Res., 4 (1989) 834.

[4] A. Van der Drift, Philips Research Report 22 (1967) 267. 
[5] This work was supported by the Director, Office of Science, Office of Basic Energy Sciences, Division of Materials Sciences and Engineering, of the U.S. Department of Energy under Contract No. DE-AC03-76SF00098.
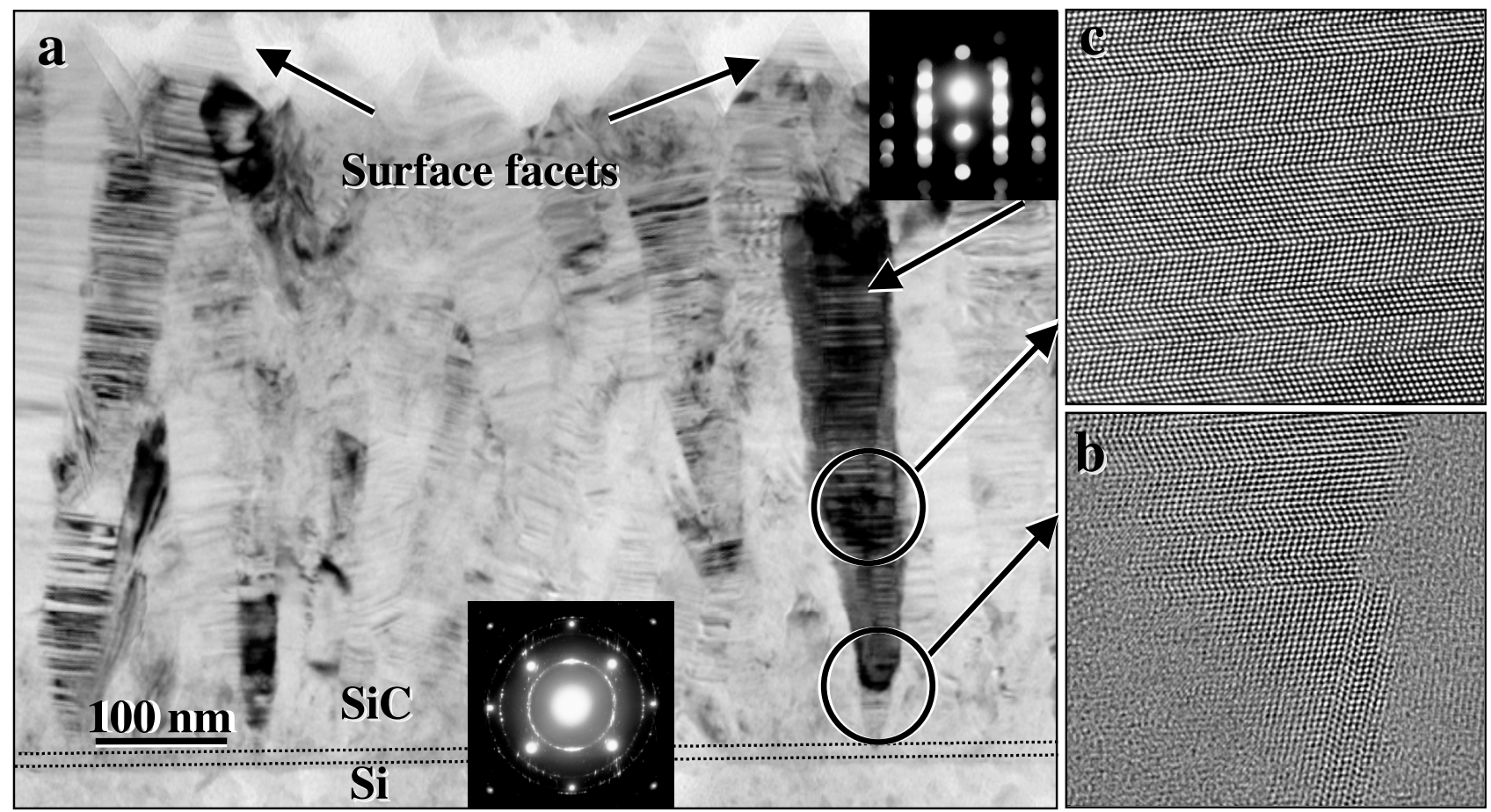

Fig. 1. a) TEM micrographs of $\mathrm{SiC}$ film in cross section demonstrating columnar growth morphology; upper right inset: nanodiffraction from arrowed grain; middle bottom inset: selected area diffraction pattern from Si substrate and SiC film; b) and c) HREM images of the encircled regions in (a); showing extensive twinning on a single set of 111 planes. From this and similar observations, an average fault density of one fault every twelve $\{111\}$ planes was measured.
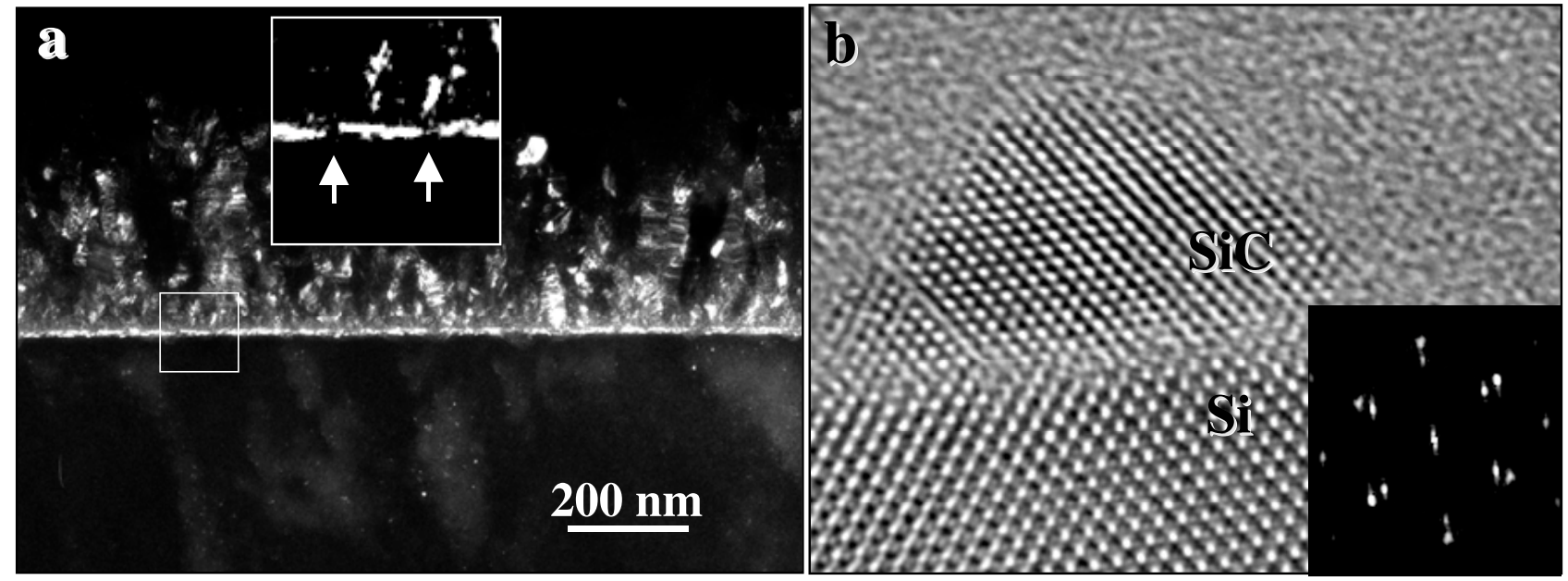

Fig. 2. a) Dark field image taken using 111 3C-SiC reflection; b) HREM micrograph showing nucleus of $\mathrm{SiC}$ on $\mathrm{Si}$ substrate in parallel-cube epitaxial orientation. The inset Fourier diffractogram indicates the orientation relationship and relative lattice parameters of $\mathrm{Si}$ and $\mathrm{SiC}$. 